\title{
Light sterile neutrinos: a critical overview
}

\author{
I. Esteban ${ }^{1^{*}}$
}

1 Departament de Fisíca Quàntica i Astrofísica and Institut de Ciencies del Cosmos, Universitat de Barcelona, Diagonal 647, E-08028 Barcelona, Spain

* ivan.esteban@fqa.ub.edu

October 26, 2018

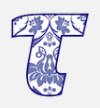

Proceedings for the 15th International Workshop on Tau Lepton Physics, Amsterdam, The Netherlands, 24-28 September 2018

scipost.org/SciPostPhysProc.Tau2018

\section{Abstract}

Light sterile neutrinos, first proposed after the results of the LSND experiment, have been a polemical topic for the last decades after seemingly contradictory data appeared from different experiments. In this overview, I review the experimental hints that point towards sterile neutrinos as well as their statistical compatibility. Even though the muon neutrino disappearance experiments strongly rule out vanilla sterile neutrinos, each oscillation channel remains internally mostly consistent. In any case, in the near future a series of independent and precise experiments should finally settle down this issue.

\section{Contents}

3

4

5

173

9

1

References
2

2

2

3

4

5

6

8

8

10 


\section{${ }_{27} 1$ Introduction}

Neutrinos are an essential part of the Standard Model of particle physics (SM). Originally, they were introduced minimally, just to explain their interactions: due to the chiral structure of the SM, only left-handed neutrinos were required. Because of that, the SM has an accidental global flavour symmetry $U(1)_{L_{e}} \times U(1)_{L_{\mu}} \times U(1)_{L_{\tau}}$ : each of the leptonic flavours $L_{\alpha}$ is separately conserved, total lepton number is conserved as well, and as a consequence neutrinos are strictly massless [1,2].

For the last decades, however, different experiments have accumulated data that conclusively shows that neutrinos change their leptonic flavour after travelling for long distances (see Ref. 3 for an overview). That is, there is conclusive evidence for new physics beyond the SM in the leptonic sector. The minimal way of explaining these flavour transitions is by giving neutrinos a mass, that, as in the quark sector, opens the possibility of flavour mixing and oscillations [4,5]. In general, for $N$ light neutrino mass eigenstates, the charged current leptonic interaction Lagrangian reads

$$
-\mathcal{L}_{\mathrm{CC}}=\frac{g}{\sqrt{2}} W_{\mu}^{+} \sum_{\substack{\alpha \in\{e, \mu, \tau\} \\ i \in\{1, \ldots, N\}}} U_{\alpha i} \bar{l}_{\alpha} \gamma_{\mu} \nu_{i}
$$

where $U$ is a $3 \times N$ mixing matrix - we know from LEP that there are only three light neutrino flavours that couple to electroweak gauge bosons [6].

As an experimental consequence, charged current interactions will produce and detect superpositions of neutrino mass eigenstates. Furthermore, if the mass eigenstates have different masses, they will evolve differently with time. Therefore, after a neutrino originally produced in a flavour $\alpha$ travels for a distance $L$, the mass eigenstates will interfere and there will be a non-zero probability of observing the neutrino in a flavour $\beta \neq \alpha$. If the neutrino beam travels in vacuum and there, this probability is given by

$$
P_{\alpha \beta}=\delta_{\alpha \beta}-4 \sum_{i<j}^{N} \operatorname{Re}\left[U_{\alpha i} U_{\beta i}^{*} U_{\alpha j}^{*} U_{\beta j}\right] \sin ^{2} \frac{\Delta m_{i j}^{2} L}{4 E}+2 \sum_{i<j}^{N} \operatorname{Im}\left[U_{\alpha i} U_{\beta i}^{*} U_{\alpha j}^{*} U_{\beta j}\right] \sin \frac{\Delta m_{i j}^{2} L}{2 E},
$$

where $E$ is the neutrino energy, $\Delta m_{i j}^{2} \equiv m_{i}^{2}-m_{j}^{2}$ is the squared-mass splitting among the light neutrino mass eigenstates.

The oscillatory dependence of Eq. 2 on $L / E$ is the smoking gun for neutrino masses as an explanation for neutrino flavour transitions. Precise spectral measurements have led to an accurate observation of two different characteristic frequencies $[7-9$. That is, numerous experiments have independently measured two different squared-mass splittings $\left(\mathcal{O}\left(10^{-3} \mathrm{eV}^{2}\right)\right.$ and $\left.\mathcal{O}\left(10^{-5} \mathrm{eV}^{2}\right)\right)$, which point to three light neutrino mass eigenstates.

\section{$2 \stackrel{(-)}{\nu}, \rightarrow \stackrel{(-)}{\nu}_{e}:$ hints towards a fourth light neutrino mass eigenstate?}

\section{$2.1 \quad$ LSND}

The so-called $3 \times 3$ paradigm ( 3 leptonic flavours and 3 light neutrino mass eigenstates) to explain the observed neutrino flavour transitions is well established. There exist, however, some discrepancies from this framework, the first of which came from the LSND experiment in the 90s [10]. This experiment had a well-understood neutrino source: a beam of protons 
63

64

hit a target, producing pions. The $\pi^{-}$were mostly absorbed, whereas the $\pi^{+}$decayed at rest

$$
\pi^{+} \rightarrow \mu^{+}+\nu_{\mu}
$$

and, finally, the $\mu^{+}$again decayed at rest

$$
\mu^{+} \rightarrow e^{+}+\nu_{e}+\bar{\nu}_{\mu}
$$

producing a very monochromatic beam of muon antineutrinos. After travelling for about $30 \mathrm{~m}$, any electron antineutrino to which the muon antineutrinos could have transitioned was detected through inverse beta decay

$$
\bar{\nu}_{e}+p \rightarrow n+e^{+} .
$$

Surprisingly, the LSND collaboration reported a $3.8 \sigma \bar{\nu}_{e}$ excess over background. This excess, shown in Fig. 1, was interpreted as due to mass-induced neutrino flavour oscillations. If this were the case, the typical LSND $L / E$ ratio requires, from Eq. 2, a squaredmass splitting $\Delta m^{2} \sim \mathcal{O}\left(\mathrm{eV}^{2}\right)$. This splitting is several orders of magnitude larger than the other two well-established ones, and therefore explaining LSND through massive neutrino oscillations requires a fourth light neutrino mass eigenstate. Due to unitarity, this means there must exist a fourth neutrino flavour eigenstate that, due to LEP constraints [6], cannot couple to the $Z$ boson. That is, if interpreted as due to neutrino oscillations, LSND points towards a sterile neutrino.

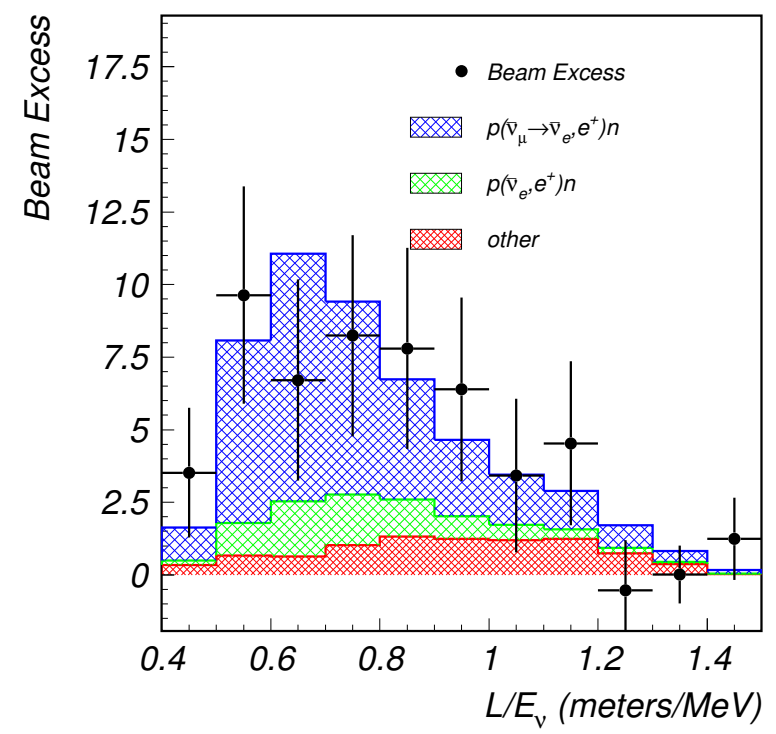

Figure 1: $\bar{\nu}_{e}$ excess over background (green) observed by the LSND experiment. The blue line corresponds to the prediction if the excess is due to neutrino masses with $\Delta m^{2} \sim \mathrm{eV}^{2}$

The LSND results are, however, rather polemical: an independent reanalysis reevaluated the neutrino fluxes, backgrounds and systematics; lowering the significance of the excess to $2.3 \sigma 11,12$.

\subsection{MiniBooNE}

2 The MiniBooNE experiment was built to independently confirm or falsify the LSND signal. ${ }_{83}$ Both production and detection were different from LSND: to produce a $\stackrel{(-)}{\nu}_{\mu}$ beam, a ${ }_{34}$ beam of protons hit a target, producing pions that decayed in flight to either $\nu_{\mu}$ or $\bar{\nu}_{\mu}$, 
depending on the experimental setup. The detector, that could detect both $\stackrel{(-)}{\nu}_{\mu}$ and $\stackrel{(-)}{\nu}_{e}$, was both a Cerenkov and scintillation detector. Furthermore, the baseline of the MiniBooNE experiment was about one order of magnitude larger than the LSND one, even though both experiments operated at similar values of $L / E$.

The results from this experiment were, however, intriguing. The observed $\nu_{e}$ and $\bar{\nu}_{e}$ spectra are shown in Fig. 2 the $\bar{\nu}_{e}$ channel shows an excess that looks quite compatible with LSND. In the $\nu_{e}$ channel, though, the excess at the $L / E$ region explored by LSND is not that significant. What is more, both channels show a low-energy excess that, particularly for the $\nu_{e}$ channel, is difficult to accommodate with mass-induced neutrino flavour oscillations (whose prediction is shown in dashed lines).

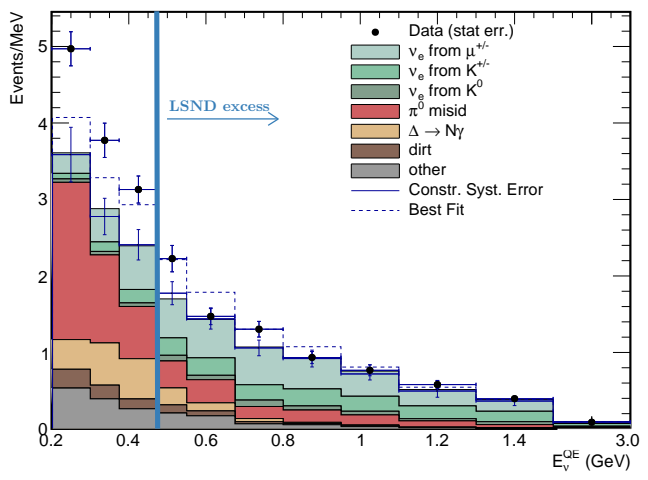

(a) $\nu_{\mu} \rightarrow \nu_{e}$

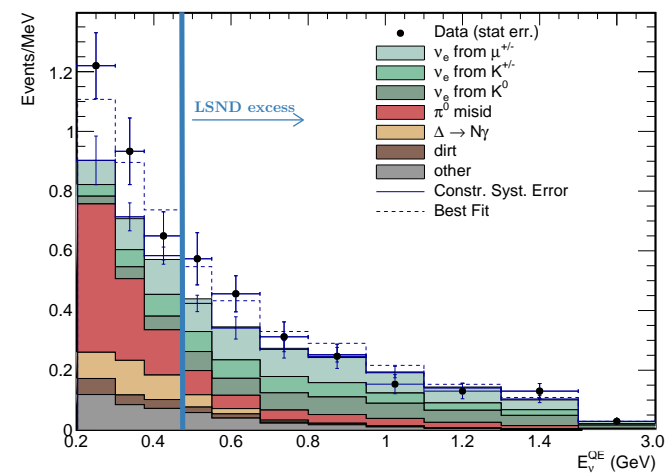

(b) $\bar{\nu}_{\mu} \rightarrow \bar{\nu}_{e}$

Figure 2: MiniBooNE excess in the $\nu_{e}$ and $\bar{\nu}_{e}$ channels. The backgrounds are shown in solid lines, whereas the best fit assuming oscillations is the dashed line. The region of $L / E$ where LSND saw the excess is located to the right of the vertical blue line.

\subsection{Combination of $\stackrel{(-)}{\nu}$ eppearance experiments}

Even though both LSND and MiniBooNE point towards neutrino flavour violation and therefore new physics, their interpretation in terms of a light sterile neutrino is unclear. On the one hand, the low-energy MiniBooNE excess does not look like oscillations at all; on the other hand, one also has to consider null results from other $\stackrel{(-)}{\nu}_{\mu} \rightarrow \stackrel{(-)}{\nu}_{e}$ experiments. A complete answer, thus, requires a global fit. Fig. 3 shows the combined result of $\stackrel{(-)}{\nu}$ appearance searches from the most recent global fit 13 . Since in the $L / E$ regime at short baselines sensitive to $\Delta m^{2} \sim \mathcal{O}\left(\mathrm{eV}^{2}\right)$ the other squared-mass splittings are negligible, the $\stackrel{(-)}{\nu}_{\mu} \rightarrow \stackrel{(-)}{\nu}_{e}$ oscillation probability can be parametrised as

$$
P\left(\stackrel{(-)}{\nu}_{\mu} \rightarrow \stackrel{(-)}{\nu}_{e}\right)=\sin ^{2}\left(2 \theta_{\mu e}\right) \sin ^{2}\left(\frac{\Delta m^{2} L}{4 E}\right)
$$

where $\sin ^{2}\left(2 \theta_{\mu e}\right)=4\left|U_{e 4}\right|^{2}\left|U_{\mu 4}\right|^{2}$, and $\Delta m^{2}=\Delta m_{41}^{2}$. The confidence regions in the global fit are shown in terms of these two parameters.

As can be seen in the figure, all the $\stackrel{(-)}{\nu}_{\mu} \rightarrow \stackrel{(-)}{\nu}_{e}$ experiments are quite consistent. The combined region, shown in red, excludes no flavour transitions with about $6 \sigma$ : the LSND and MiniBooNE excesses are not a statistical fluctuation. The goodness-of-fit, though, is not that good: Ref. 13 finds $\chi_{\min }^{2} /$ dof $=89.9 /(69-2)$, which corresponds to a $p$-value

\footnotetext{
${ }^{1}$ It does not include the latest MiniBooNE results, but the qualitative conclusions should not change.
} 


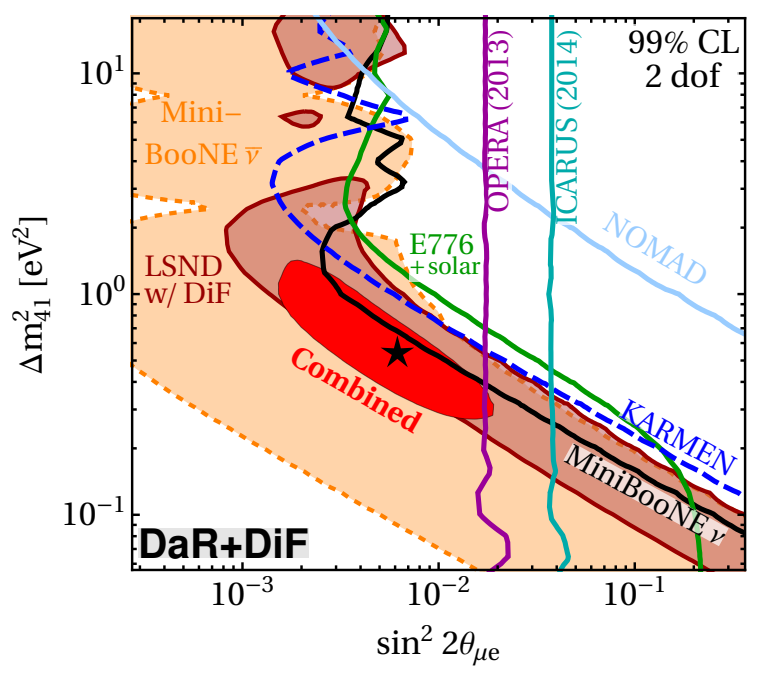

Figure 3: Combined $\stackrel{(-)}{\nu}_{\mu} \rightarrow \stackrel{(-)}{\nu}_{e}$ results. All the parameter space inside the coloured regions is allowed at $99 \%$ CL by the two experiments that saw a positive signal: LSND and MiniBooNE. All the parameter space to the right of the coloured lines is disfavoured at $99 \%$ CL by experiments that did not see any significant excess above background. The regions are shown in the $\Delta m^{2}$ vs $\sin ^{2}\left(2 \theta_{\mu e}\right)$ plane: see text for details. Figure extracted from Ref. [13].

of $3 \%$. This is mostly driven by the MiniBooNE low-energy excess, that as has been mentioned, is rather difficult to fit with oscillations.

\section{$3 \stackrel{(-)}{\nu}_{e} \rightarrow \stackrel{(-)}{\nu}_{e}$ disappearance}

If the LSND and MiniBooNE signals were due to an eV-scale sterile neutrino, we would not only see neutrino flavour violation in $\stackrel{(-)}{\nu}_{\mu} \rightarrow \stackrel{(-)}{\nu}_{e}$. Instead, there should also be signals in many other short-baseline experiments. And, in fact, another hint in favour of light sterile neutrinos comes from short-baseline reactor experiments.

These hints first started to appear after Refs. [14, 15] re-evaluated the $\bar{\nu}_{e}$ fluxes from nuclear reactors. Using more sophisticated ab-initio calculations and more modern data, they found that the theoretical flux was being overestimated by a factor of about $3 \%$. That is, all the short-baseline reactor experiments, that were not seeing any unusual result, were actually seeing a $3 \% \bar{\nu}_{e}$ deficit. Fig. 4 shows the measured flux normalisation from shortbaseline reactor experiments before the re-evaluation: all data points consistently sit below the most recent prediction.

This $\bar{\nu}_{e}$ deficit can be attributed to short-baseline flavour oscillations. Intriguingly, the $L / E$ ratio points towards $\Delta m^{2} \sim \mathcal{O}\left(\mathrm{eV}^{2}\right)$, consistently with the LSND and MiniBooNE signals. However, the reactor experiments in Fig. 4 did not have enough energy resolution to disentangle the $\sin ^{2} \frac{\Delta m^{2} L}{4 E}$ modulation of the transition amplitude, which is the true smoking gun for neutrino oscillations. Instead, they could only see the averaged effect, and so this reactor antineutrino anomaly might as well be due to flux mismodellings.

In fact, there are some other experimental hints pointing towards theoretical errors. The RENO experiment first reported a bump in their spectrum, at a neutrino energy $\sim 5 \mathrm{MeV}$, that was not predicted by the theoretical calculations [16]. The bump is shown in Fig. 5 as accurately measured by the RENO and Daya Bay experiments. Being a $\bar{\nu}_{e}$ 


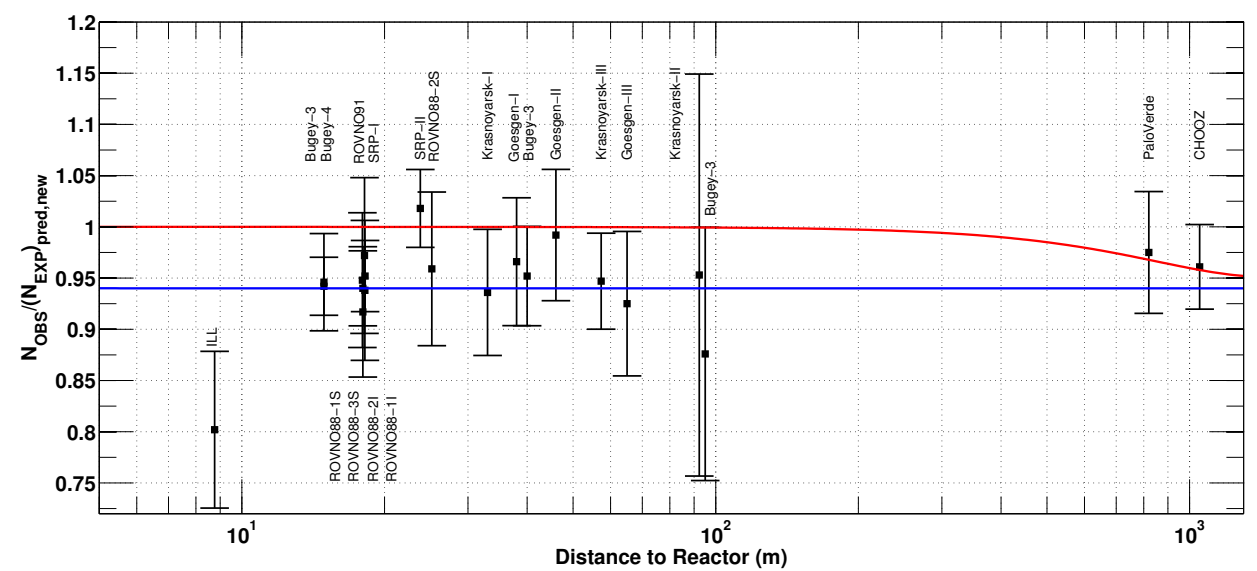

Figure 4: Reactor neutrino flux normalisation as seen in different experiments before the re-evaluation of the fluxes in Refs. [14, 15]. The blue line is the old prediction, the red line is the new one (including the effect due to non-zero $\theta_{13}$ ). More recent experiments have consistently measured fluxes in accordance with the old calculations.

excess, and not a deficit, it cannot be explained by simple oscillations (even though there are some exotic explanations, see Ref. [17]). Therefore, it sheds some doubt on the validity of the nuclear physics calculations that led to the interpretation of the deficit discussed above as due to sterile neutrinos.

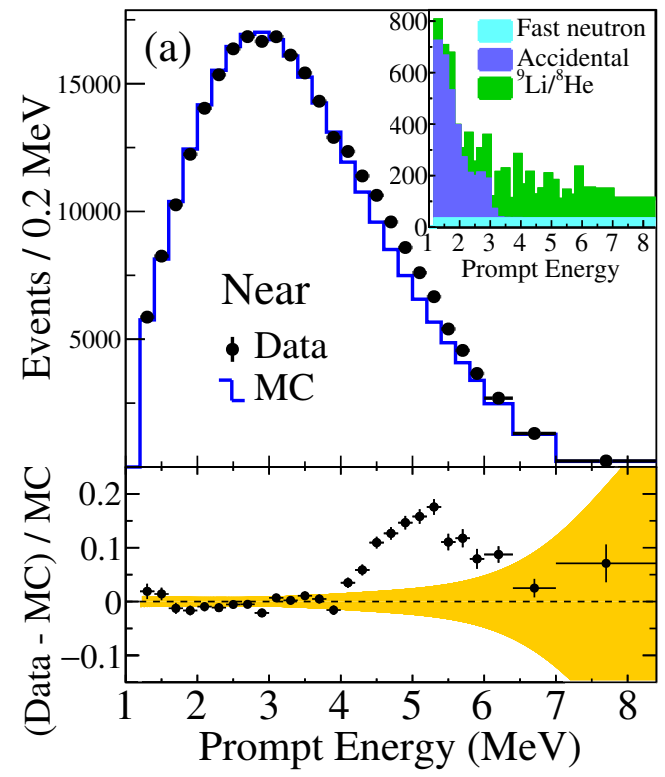

(a) RENO

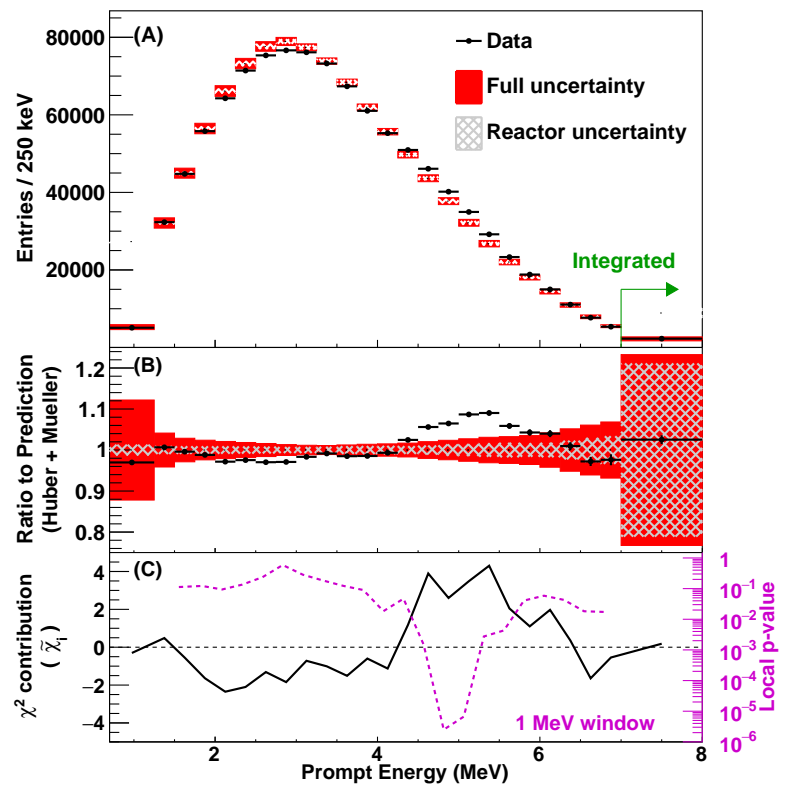

(b) Daya Bay

Figure 5: $5 \mathrm{MeV}$ bump as measured by the RENO [16] and Daya Bay [18] collaborations.

\subsection{Sterile neutrinos?}

The interpretation of the reactor antineutrino anomaly as due to sterile neutrinos is supported by some results that are independent of flux calculations. In particular, there are currently two experiments with published data, NEOS [19] and DANSS [20], that have 


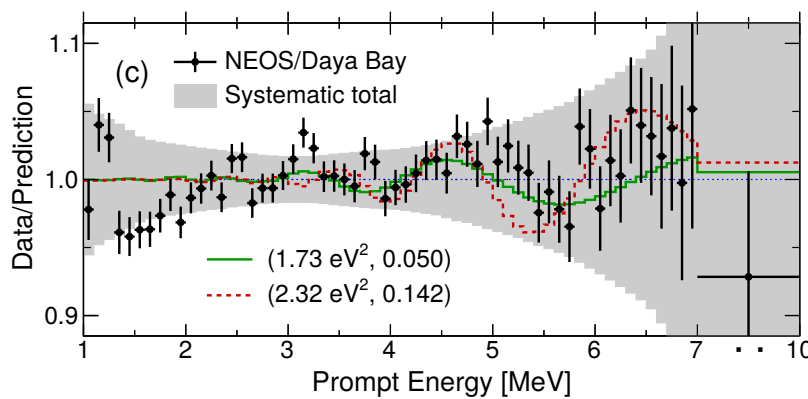

(a) NEOS he $L$-dependence of the transition probability. have enough statistical significance yet.

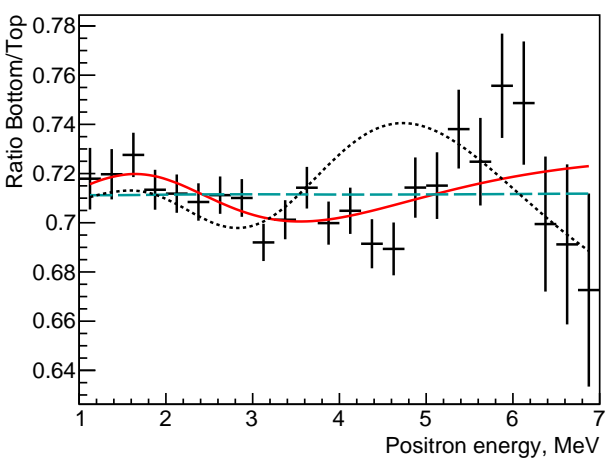

(b) DANSS

Figure 6: Reactor antineutrino spectra measured by the NEOS 19 and DANSS 20 experiments. Both of them see an energy-dependent flavor transition probability that can be fitted by neutrino oscillations, independently of any theoretical flux calculation.

enough energy resolution to disentangle the $L / E$ modulation of the transition probability. The NEOS experiment does it by comparing their results to the experimental flux measured with great accuracy by Daya Bay. The DANSS experiment, on the other hand, has a movable detector that sits close to the nuclear reactor, and they can therefore measure

Interestingly, both experiments report some "wiggles" in their spectra that can be fitted by sterile neutrino oscillations. Nevertheless, the results, shown in Fig. 6, do not

Apart from these results, there were also a set of experiments that reported $\nu_{e}$ disappearance at short baselines from neutrino sources other than nuclear reactors. In particular, the GALLEX [21] and SAGE [22,23 experiments measured the ${ }^{71} \mathrm{Ga} \nu_{e}$ capture cross section with neutrinos coming from intense radioactive sources. They consistently measured a cross section below the theoretical prediction (see Fig. 7), which can be interpreted as due to short-baseline neutrino oscillations. Since the neutrinos came from a radioactive source, these results are completely independent of any theoretical reactor flux calculation.

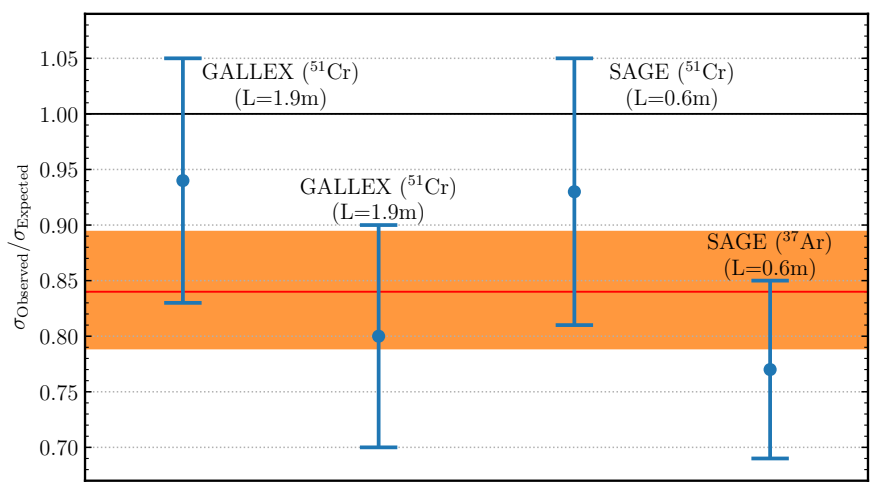

Figure 7: The ${ }^{71} \mathrm{Ga} \nu_{e}$ capture cross section as measured by the GALLEX [21] and SAGE 22, 23] experiments at short baselines $L$, with neutrinos coming from different radioactive sources (in parentheses). All the data points consistently sit below the theoretical prediction (in black): the measured average and its error are shown in red and orange, respectively. 


\subsection{Or nuclear physics miscalculations?}

Despite the evidences presented in the previous section, there are also experimental results that support flux miscalculations as the origin of the reactor antineutrino anomaly. In more detail, both the Daya Bay [24] and RENO [25] experiments were able to monitor the abundance of the two most common $\beta$-decaying isotopes in their nuclear reactors: ${ }^{239} \mathrm{Pu}$ and ${ }^{235} \mathrm{U}$. Therefore, they could disentangle whether the observed flux deficit affects both isotopes in the same way - the expected result if it were due to sterile neutrino oscillations - or not.

Their results are shown in Fig. 8, where they show the yield (defined as the number of events over the flux) both for ${ }^{239} \mathrm{Pu}$ and ${ }^{235} \mathrm{U}$. Interestingly, their flux measurements for ${ }^{239} \mathrm{Pu}$ agree with the theoretical expectations from Refs. 14, 15, whereas the ${ }^{235} \mathrm{U}$ measurements show a deficit with respect to expectations. That is, the results point towards ${ }^{235} \mathrm{U}$ as the source of the reactor antineutrino anomaly. In principle, this rules out sterile neutrinos as en explanation, because they should affect both isotopes in the same way.

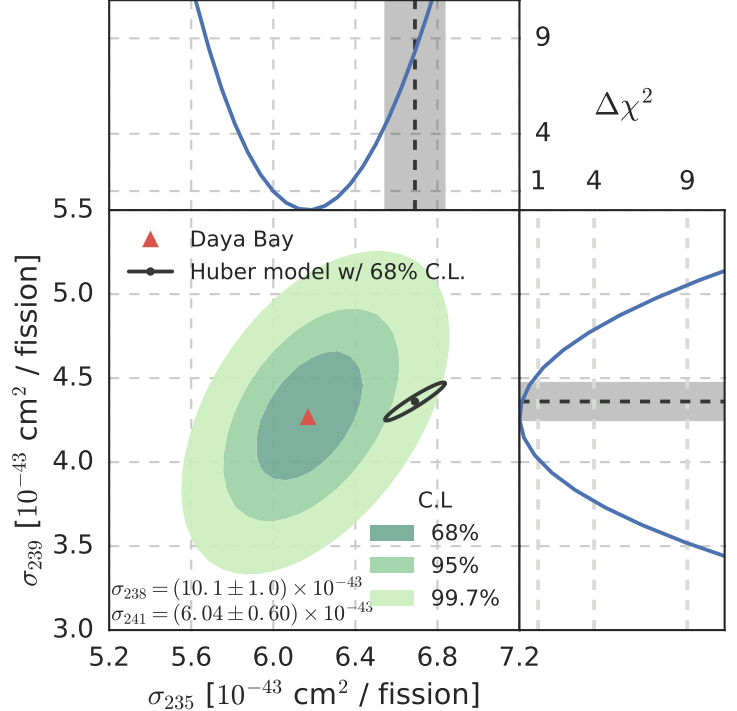

(a) Daya Bay

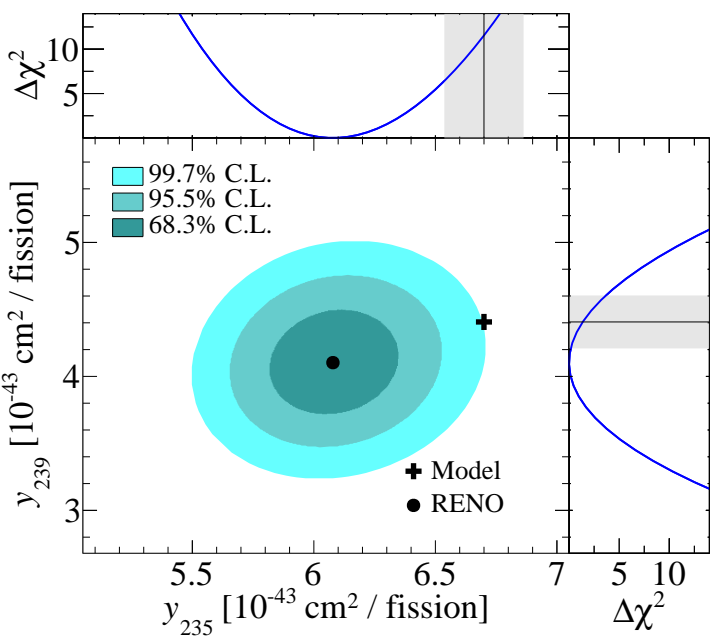

(b) RENO

Figure 8: Observed flux yields for ${ }^{239} \mathrm{Pu}$, in the vertical axis, and ${ }^{235} \mathrm{U}$, in the horizontal axis. Theoretical expectations are shown in black (left) and with a cross (right). The ${ }^{239} \mathrm{Pu}$ measurement agrees with the theoretical expectations, whereas ${ }^{235} \mathrm{U}$ shows a deficit. Figures extracted from Refs. 24,25].

What is more, in Ref. [25], the RENO collaboration showed that the relative amplitude of the $5 \mathrm{MeV}$ bump is correlated with the amount of ${ }^{235} \mathrm{U}$ in their nuclear reactor. These results again shed doubt on the validity of the theoretical flux calculations for this isotope.

\subsection{Combination of $\stackrel{(-)}{\nu}$ disappearance experiments}

The seemingly contradictory results regarding the origin of the reactor antineutrino anomaly call for a global fit that assesses the compatibility among different data sets. Regarding just reactor results, Ref. [13] combined all the data available in March 2018 under two different hypotheses: they either assumed the theoretical reactor flux calculations from Refs. 14, 15, or they left the global normalisation of the reactor flux free (therefore assuming nuclear physics miscalculations). 
The results are shown in Fig. 9. Interestingly, both for free and fixed flux normalisations, the 95\% CL region points towards the presence of sterile neutrino oscillations. To numerically quantify the statistical significance of each hypothesis, Tab. 1 shows the minimum $\chi^{2}$ for either neutrino oscillations or not, and either free or fixed fluxes. Currently, the hypothesis that best describes the data is oscillations and a free flux normalisation: this hypothesis can accommodate both the oscillatory patterns seen by NEOS and DANSS, as well as the isotope-dependent flux measurements. Nevertheless, oscillations and fixed fluxes (i.e., a pure sterile neutrino solution for the reactor antineutrino anomaly) is basically at the same level of confidence as no oscillations and free fluxes (i.e., nuclear physics miscalculations as the only source of the reactor antineutrino anomaly): we still need more data to disentangle this issue.

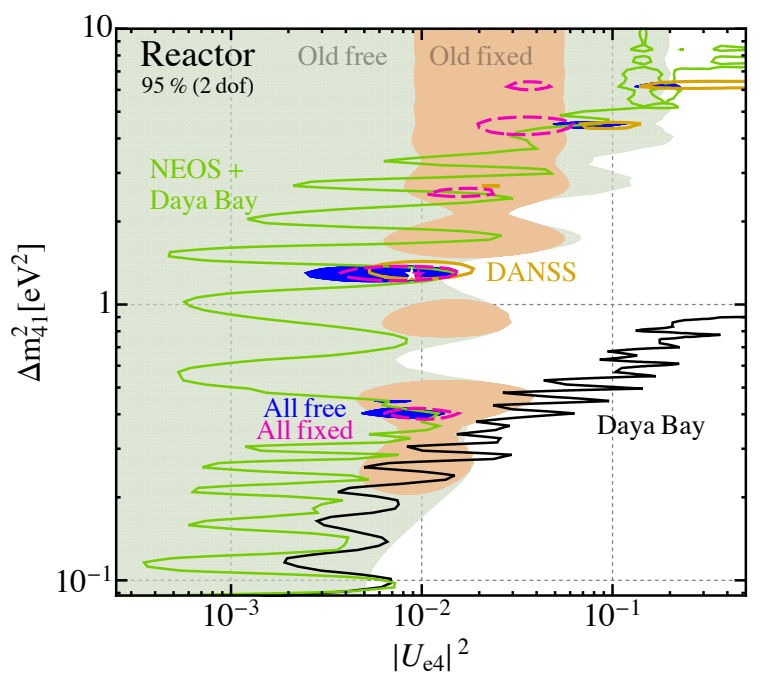

Figure 9: Combined $\bar{\nu}_{e}$ disappearance results from reactors. All the parameter space inside the coloured regions is allowed at $95 \% \mathrm{CL}$, and the parameter space to the right of the lines is disallowed with the same confidence level. The global combination is shown in blue for free flux normalisation, and in pink for fixed theoretical flux calculations. Figure extracted from Ref. [13].

\begin{tabular}{cc} 
Hypothesis & $\chi_{\min }^{2}$ \\
\hline Oscillations + free fluxes & 185.8 \\
Oscillations + fixed fluxes & 196.0 \\
No oscillations + free fluxes & 197.3 \\
No oscillations + fixed fluxed & 211.5
\end{tabular}

Table 1: $\chi_{\min }^{2}$ either assuming neutrino oscillations or not, and free or fixed fluxes. Data extracted from Ref. [13].

The reactor data can also be combined with the ${ }^{71} \mathrm{Ga}$ anomaly data, as well as with null searches. The combined regions are shown in Fig. 10, no neutrino oscillations are disfavoured by $3.2 \sigma(3.8 \sigma)$ for free (fixed) reactor fluxes. There is a minor tension between reactor and ${ }^{71} \mathrm{Ga}$ data, though: the $p$-value for compatibility is $9 \%$, or even $3 \%$ once the data from all the other $\stackrel{(-)}{\nu}_{e}$ disappearance experiments is included. 


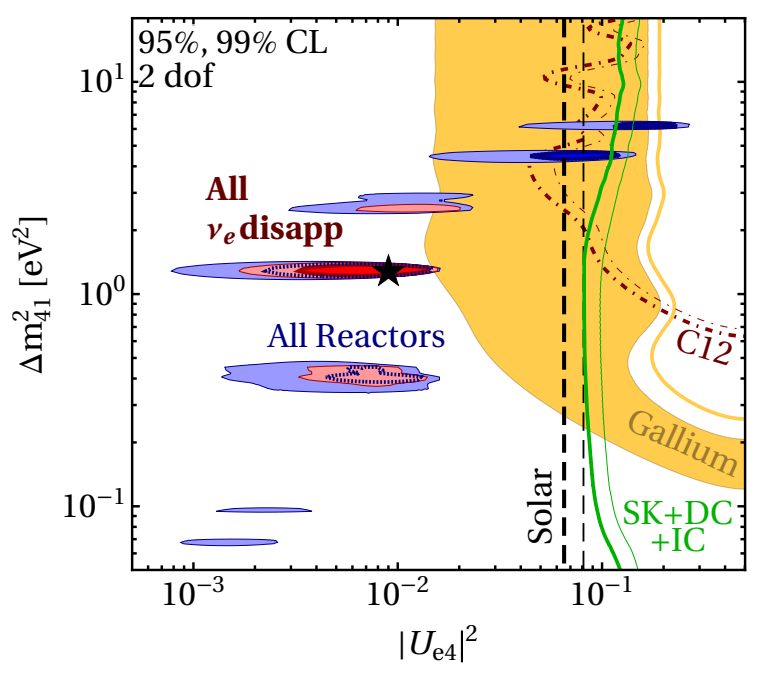

Figure 10: Combined $\stackrel{(-)}{\nu}$ e disappearance results, assumming free reactor flux normalisation. All the parameter space inside the coloured regions is allowed, and the parameter space to the right of the solid lines disallowed. Figure extracted from Ref. [13].

\section{The appearance-disappearance tension and other issues}

As shown in the previous sections, there are hints towards the existence of a fourth light neutrino mass eigenstate, coming both from $\stackrel{(-)}{\nu}_{\mu} \rightarrow \stackrel{(-)}{\nu}_{e}$ and from $\stackrel{(-)}{\nu}_{e} \rightarrow \stackrel{(-)}{\nu}_{e}$ experiments. Despite some minor internal tensions, they all point in the same direction: a fourth neutrino mass eigenstate with $\Delta m_{41}^{2} \sim \mathcal{O}\left(\mathrm{eV}^{2}\right)$, which induces $\stackrel{(-)}{\nu}_{\mu} \rightarrow \stackrel{(-)}{\nu}_{e}$ transitions with an amplitude $4\left|U_{e 4}\right|^{2}\left|U_{\mu 4}\right|^{2} \sim 6 \cdot 10^{-3}$, and $\left.\stackrel{(-)}{\nu}, \rightarrow \stackrel{(-)}{\nu}\right)$ transitions with an amplitude $\left|U_{e 4}\right|^{2} \sim 10^{-2}$.

Therefore, if the picture is consistent, we should also see $\stackrel{(-)}{\nu}_{\mu}$ disappearance with an amplitude $\left|U_{\mu 4}\right|^{2} \sim 10^{-1}$. And we do not: Fig. 11 shows the combination of constraints coming from different experiments, that clearly rules out the region allowed by all the data discussed in the previous sections. The global fit in Ref. [13] gives a very low $p$ value for compatibility of $3.71 \cdot 10^{-7}$ : vanilla sterile neutrinos as an explanation for the anomalies mentioned in Sections 2 and 3 are ruled out with $4.7 \sigma$. This problem is called the appearance-disappearance tension.

What is more, the fit does not significantly improve if one single experiment is removed. Not even if we remove all reactor and gallium data, that points towards $\left|U_{e 4}\right|^{2} \sim 10^{-2}$ and therefore gives the required value of $\left|U_{\mu 4}\right|^{2}$ to explain LSND and MiniBooNE, does the fit significantly improve.

On top of that, vanilla sterile neutrinos also have severe issues with cosmology. If they are thermally produced in the early universe via mixing, they would increase the relativistic energy content of the universe. The effective number of relativistic degrees of freedom, however, is bound to be 26

$$
\left.N_{\text {eff }}=3.1 \pm 0.3 \quad \text { (95\%C.L. }\right),
$$

far from $N_{\text {eff }} \sim 4$, the prediction if there is a fourth light neutrino mass eigenstate. Furthermore, massive neutrinos have an imprint on the gravitational potential, and combining $\mathrm{CMB}$ and $\mathrm{BAO}$ data we get the following bound on the sum of neutrino masses [26]:

$$
\sum m_{\nu}<0.12 \mathrm{eV} \quad(95 \% \text { C.L. }),
$$




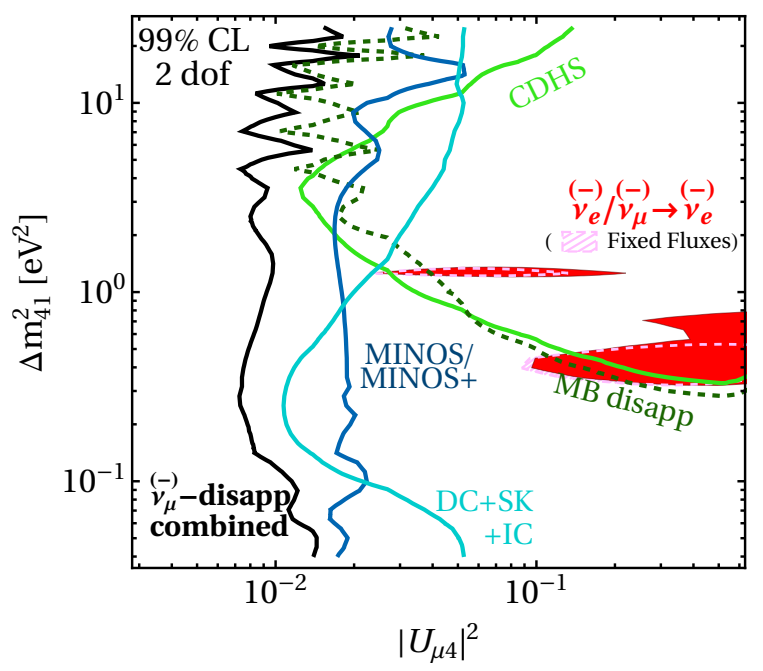

Figure 11: Constraints coming from not observing $\stackrel{(-)}{\nu}$, disappearance driven by a fourth light neutrino mass eigenstate. All the parameter space to the right of the solid lines is ruled out with $95 \%$ CL. The region preferred at that CL by the $\stackrel{(-)}{\nu}_{\mu} \rightarrow \stackrel{(-)}{\nu}_{e}$ and $\left.\stackrel{(-)}{\nu}\right) \rightarrow \stackrel{(-)}{\nu}_{e}$ data is shown in red. Figure extracted from Ref. [13].

which is hard to reconcile with an $\mathrm{eV}$-scale neutrino mass eigenstate.

Of course, the bounds, coming from cosmology, are indirect. In fact, Refs. [27, 28 proposed a way to avoid the cosmological bounds by charging the sterile flavour eigenstate under a new gauge interaction that would suppress the production of the heavy neutrino mass eigenstate in the early universe. These models, however, are currently ruled out 29 , 30.

All in all, models that are free of the appearance-disappearance tension as well as cosmological constraints need to introduce some other new physics in addition to a light sterile neutrino. There are for instance, models that are able to explain the MiniBooNE low energy excess with a very small $\left|U_{\mu 4}\right|^{2}$, and which could also have observable consequences in other experiments [31,32].

\subsection{Future prospects}

Be that as it may, the final word on the existence of light sterile neutrinos will only come from the experiment. Regarding the $\stackrel{(-)}{\nu}_{\mu} \rightarrow \stackrel{(-)}{\nu}_{e}$ sector, there is a strong short-baseline programme at Fermilab 33 that will start releasing data around 2019. It uses the same beam as MiniBooNE and should be able to confirm or definitely rule out the LSND/MiniBooNE excess. If it is really there, its modern detectors will allow to investigate its origin; and furthermore they have a near detector, unlike MiniBooNE, to better calibrate their backgrounds.

In the $\stackrel{(-)}{\nu}_{e}$ disappearance sector, there are many reactor and non-reactor experiments that will continue releasing baseline-dependent and isotope dependent data. If there is a light sterile neutrino behind the reactor antineutrino anomaly, its $L / E$ modulation should definitely show up in the following years. 


\section{Conclusion}

There are several hints from neutrino appearance and disappearance experiments at short baselines that point towards new physics in the leptonic sector. These hints come from leptonic flavour violation, a clear signal for physics beyond the Standard Model, with both neutrinos and antineutrinos, different sources, and different detection techniques. They have been mostly interpreted as being due to light sterile neutrinos, even though some of the hints are subject to possible nuclear mismodellings and even though there is a severe appearance-disappearance tension. Along with cosmological constraints, the tensions strongly rule out vanilla sterile neutrinos as an explanation for the anomalies. In any case, in the near future a set of independent and precise experiments will soon settle down this issue, and will point either to uncontrolled experimental uncertainties or to some other exotic new physics.

\section{Acknowledgements}

I would like to thank the organisers of the conference for their invitation and kind hospitality. I would also like to acknowledge Joachim Kopp for helpful discussions.

Funding information This work is funded by the FPU program fellowship of the Spanish Ministry of Education, Culture and Sports, FPU15/03697.

\section{References}

[1] B. Pontecorvo, Neutrino Experiments and the Problem of Conservation of Leptonic Charge, Sov. Phys. JETP 26, 984 (1968), [Zh. Eksp. Teor. Fiz.53,1717(1967)].

[2] V. N. Gribov and B. Pontecorvo, Neutrino astronomy and lepton charge, Phys. Lett. 28B, 493 (1969), doi:10.1016/0370-2693(69)90525-5.

[3] M. C. Gonzalez-Garcia and M. Maltoni, Phenomenology with Massive Neutrinos, Phys. Rept. 460, 1 (2008), doi:10.1016/j.physrep.2007.12.004, 0704.1800

[4] Z. Maki, M. Nakagawa and S. Sakata, Remarks on the unified model of elementary particles, Prog. Theor. Phys. 28, 870 (1962), doi:10.1143/PTP.28.870, [,34(1962)].

[5] M. Kobayashi and T. Maskawa, CP Violation in the Renormalizable Theory of Weak Interaction, Prog. Theor. Phys. 49, 652 (1973), doi:10.1143/PTP.49.652.

[6] S. Schael et al., Precision electroweak measurements on the $Z$ resonance, Phys. Rept. 427, 257 (2006), doi:10.1016/j.physrep.2005.12.006, hep-ex/0509008.

[7] I. Esteban, M. C. Gonzalez-Garcia, M. Maltoni, I. Martinez-Soler and T. Schwetz, Updated fit to three neutrino mixing: exploring the accelerator-reactor complementarity, JHEP 01, 087 (2017), doi:10.1007/JHEP01(2017)087, 1611.01514.

[8] F. Capozzi, E. Lisi, A. Marrone, D. Montanino and A. Palazzo, Neutrino masses and mixings: Status of known and unknown $3 \nu$ parameters, Nucl. Phys. B908, 218 (2016), doi:10.1016/j.nuclphysb.2016.02.016, 1601.07777. 
[9] P. F. de Salas, D. V. Forero, C. A. Ternes, M. Tortola and J. W. F. Valle, Status of neutrino oscillations 2018: $3 \sigma$ hint for normal mass ordering and improved $C P$ sensitivity, Phys. Lett. B782, 633 (2018), doi:10.1016/j.physletb.2018.06.019, 1708. 01186 .

[10] A. Aguilar-Arevalo et al., Evidence for neutrino oscillations from the observation of anti-neutrino(electron) appearance in a anti-neutrino(muon) beam, Phys. Rev. D64, 112007 (2001), doi:10.1103/PhysRevD.64.112007, hep-ex/0104049.

[11] A. Bolshakova et al., Revisiting the 'LSND anomaly' I: impact of new data, Phys. Rev. D85, 092008 (2012), doi:10.1103/PhysRevD.85.092008, 1110.4265.

[12] A. Bolshakova et al., Revisiting the 'LSND anomaly' II: critique of the data analysis, Phys. Rev. D85, 092009 (2012), doi:10.1103/PhysRevD.85.092009, 1112.0907.

[13] M. Dentler, A. Hernandez-Cabezudo, J. Kopp, M. Maltoni and T. Schwetz, Sterile neutrinos or flux uncertainties? - Status of the reactor anti-neutrino anomaly, JHEP 11, 099 (2017), doi:10.1007/JHEP11(2017)099, 1709.04294.

[14] T. A. Mueller et al., Improved Predictions of Reactor Antineutrino Spectra, Phys. Rev. C83, 054615 (2011), doi:10.1103/PhysRevC.83.054615, 1101.2663.

[15] P. Huber, On the determination of anti-neutrino spectra from nuclear reactors, Phys. Rev. C84, 024617 (2011), doi:10.1103/PhysRevC.85.029901, 10.1103/PhysRevC.84.024617, [Erratum: Phys. Rev.C85,029901(2012)], 1106.0687.

[16] J. H. Choi et al., Observation of Energy and Baseline Dependent Reactor Antineutrino Disappearance in the RENO Experiment, Phys. Rev. Lett. 116(21), 211801 (2016), doi:10.1103/PhysRevLett.116.211801, 1511.05849

[17] J. M. Berryman, V. Brdar and P. Huber, Nuclear and Particle Conspiracy Solves Both Reactor Antineutrino Anomalies (2018), 1803.08506.

[18] F. P. An et al., Improved Measurement of the Reactor Antineutrino Flux and Spectrum at Daya Bay, Chin. Phys. C41(1), 013002 (2017), doi:10.1088/1674$1137 / 41 / 1 / 013002,1607.05378$.

[19] Y. Ko et al., Sterile Neutrino Search at the NEOS Experiment, Phys. Rev. Lett. 118(12), 121802 (2017), doi:10.1103/PhysRevLett.118.121802, 1610.05134.

[20] I. Alekseev et al., Search for sterile neutrinos at the DANSS experiment (2018), 1804.04046 .

[21] F. Kaether, W. Hampel, G. Heusser, J. Kiko and T. Kirsten, Reanalysis of the GALLEX solar neutrino flux and source experiments, Phys. Lett. B685, 47 (2010), doi:10.1016/j.physletb.2010.01.030, 1001.2731.

[22] J. N. Abdurashitov et al., Measurement of the response of the Russian-American gallium experiment to neutrinos from a Cr-51 source, Phys. Rev. C59, 2246 (1999), doi:10.1103/PhysRevC.59.2246, hep-ph/9803418.

[23] J. N. Abdurashitov et al., Measurement of the response of a Ga solar neutrino experiment to neutrinos from an Ar-37 source, Phys. Rev. C73, 045805 (2006), doi:10.1103/PhysRevC.73.045805, nucl-ex/0512041. 
323

[24] F. P. An et al., Evolution of the Reactor Antineutrino Flux and Spectrum at Daya Bay, Phys. Rev. Lett. 118(25), 251801 (2017), doi:10.1103/PhysRevLett.118.251801, 1704.01082 .

[25] G. Bak et al., Fuel-composition dependent reactor antineutrino yield and spectrum at RENO (2018), 1806.00574.

[26] N. Aghanim et al., Planck 2018 results. VI. Cosmological parameters (2018), 1807. 06209 .

[27] S. Hannestad, R. S. Hansen and T. Tram, How Self-Interactions can Reconcile Sterile Neutrinos with Cosmology, Phys. Rev. Lett. 112(3), 031802 (2014), doi:10.1103/PhysRevLett.112.031802, 1310.5926.

[28] B. Dasgupta and J. Kopp, Cosmologically Safe eV-Scale Sterile Neutrinos and Improved Dark Matter Structure, Phys. Rev. Lett. 112(3), 031803 (2014), doi:10.1103/PhysRevLett.112.031803, 1310.6337.

[29] N. Song, M. C. Gonzalez-Garcia and J. Salvado, Cosmological constraints with selfinteracting sterile neutrinos (2018), 1805.08218 .

[30] X. Chu, B. Dasgupta, M. Dentler, J. Kopp and N. Saviano, Sterile Neutrinos with Secret Interactions - Cosmological Discord? (2018), 1806.10629.

[31] E. Bertuzzo, S. Jana, P. A. N. Machado and R. Zukanovich Funchal, A Dark Neutrino Portal to Explain MiniBooNE (2018), 1807.09877.

[32] P. Ballett, S. Pascoli and M. Ross-Lonergan, U(1)' mediated decays of heavy sterile neutrinos in MiniBooNE (2018), 1808.02915.

[33] M. Antonello et al., A Proposal for a Three Detector Short-Baseline Neutrino Oscillation Program in the Fermilab Booster Neutrino Beam (2015), 1503.01520. 